See Article page 144 .

\section{Commentary: Mechanical circulatory support for the failing Glenn circulation: Keep all options on the table and don't give up}

\author{
Nicholas D. Andersen, MD, \\ Douglas M. Overbey, MD, and \\ Joseph W. Turek, MD, PhD
}

Mechanical circulatory support (MCS) in children is hard, MCS in single-ventricle children is harder, and MCS support of the stage 2 (Glenn) circulation is perhaps the most challenging and unpredictable anatomic and physiologic state to support with MCS therapy. In this issue of the Journal, Bedzra and colleagues ${ }^{1}$ provide a useful case report on MCS support of the Glenn circulation in a patient with postcardiotomy failure following a high-risk bilateral Glenn/Damus-Kaye-Stansel (DKS) operation. The patient was supported for $>1$ year with a 2-cannula (DKS/apical) Berlin heart configuration, augmented with separate extracorporeal membrane oxygenation (ECMO) cannulation (innominate artery/right atrium) for a brief period due to hypoxia.

This case highlights the unpredictability of the failing Glenn circulation and the need to design a customized, flexible plan for each patient on a case-by-case basis. Options for MCS in Glenn patients may include pulsatile or centrifugal support devices with or without the use of an oxygenator, preservation of the Glenn, takedown of the Glenn and placement of an aortopulmonary shunt, or

\footnotetext{
From the Section of Pediatric Cardiac Surgery, Division of Cardiovascular and Thoracic Surgery, Department of Surgery, Duke University Medical Center, Durham, NC.

Disclosures: The authors reported no conflicts of interest.

The Journal policy requires editors and reviewers to disclose conflicts of interest and to decline handling or reviewing manuscripts for which they may have a conflict of interest. The editors and reviewers of this article have no conflicts of interest.

Received for publication Dec 7, 2020; revisions received Dec 7, 2020; accepted for publication Dec 18, 2020; available ahead of print Dec 25, 2020.

Address for reprints: Nicholas D. Andersen, MD, Section of Pediatric Cardiac Surgery, Division of Cardiovascular and Thoracic Surgery, Department of Surgery, Duke University Medical Center, 2301 Erwin Rd, DUMC 3474, Durham, NC 27710 (E-mail: Nicholas.Andersen@Duke.edu)

JTCVS Techniques 2021;6:149-50

2666-2507

Copyright (C) 2020 The Authors. Published by Elsevier Inc. on behalf of The American Association for Thoracic Surgery. This is an open access article under the CC BY-NCND license (http://creativecommons.org/licenses/by-nc-nd/4.0/).

https://doi.org/10.1016/j.xjtc.2020.12.010
}

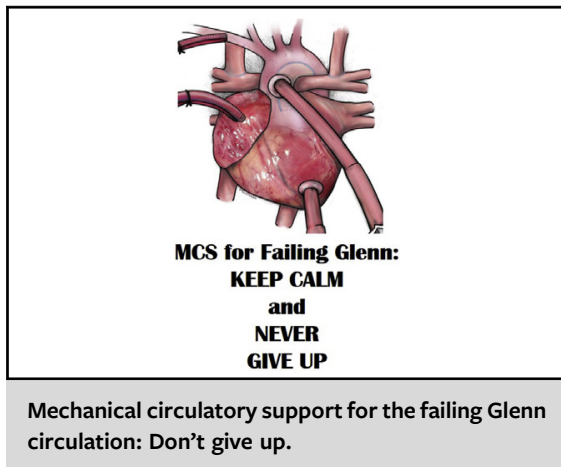

CENTRAL MESSAGE

Successful mechanical circulatory support for failing Glenn circulation requires a customized and flexible approach tailored to the dynamic anatomic and physiologic needs of each patient.

Fontan completion., ${ }^{2,3}$ The patient's specific mode of cardiopulmonary failure and anatomic complexities of the patient, as well as the strengths of the individual heart failure program factor into the decision matrix on how to proceed. In the present case, the mode of failure appeared to be related primarily to ventricular dysfunction, and the Glenn/pulmonary circuit proved adequate for long-term oxygenation of the patient. The need for ECMO support was temporary and was thought to be related to a transient increase in pulmonary vascular resistance (PVR) related to cardiopulmonary bypass after ventricular assist device conversion. However, in other patients, a fundamental anatomic problem with the Glenn connections, decompressing venous collaterals, or irreversibly high PVR may mandate taking down either one or both Glenn connections and/or maintenance of an oxygenator within the circuit for the duration of MCS support. If long-term oxygenator support were required, conversion from a Berlin to a centrifugal pump likely would be more practical.

Perhaps the most remarkable aspect of this case is that the patient endured multiple operations and was managed with MCS for $>1$ year without significant neurologic injury. Given the poor outcomes after cardiopulmonary resuscitation in patients with Glenn physiology, the aggressive use of MCS from the first signs of trouble, and the courage to consider long-term MCS support as a bridge to transplantation appear to have served this patient well. This also speaks 
to the poise and discipline of the MCS program at this hospital, because the physicians and caretakers were willing to take on this risk, remained flexible to the changing physiology, and tailored the plan to the evolving needs of the patient. Although MCS survival in Glenn patients is relatively poor, the few successes reported in the literature were likely the result of this exact approach. Stay calm, stay flexible, design a tailored approach specific to the patient, and don't give up.

\section{References}

1. Bedzra EKS, Barnes A, Birnbaum B, St. Louis JD. Mechanical support of superior cavopulmonary (Glenn) physiology to heart transplantation. J Thorac Cardiovasc Surg Tech. 2021;6:144-6.

2. Maeda K, Nasirov T, Yarlagadda V, Hollander SA, Navaratnam M, Rosenthal DN, et al. Single ventricular assist device support for the failing bidirectional Glenn patient. Ann Thorac Surg. 2020;110:1659-66.

3. Adachi I, Williams E, Jeewa A, Elias B, McKenzie ED. Mechanically assisted Fontan completion: a new approach for the failing Glenn circulation due to isolated ventricular dysfunction. J Heart Lung Transplant. 2016;35:1380-1. 